\title{
Das Bundesgericht weist die Filmverleiher in die Urheberrechtsschranken
}

Bemerkungen zum Urteil des Bundesgerichts 4A_433/2018 (BGE 145 III 72)

Matthias Schwaibold *

Die Swisscom als blosser Access Provider kann nicht verpflichtet werden, den Zugriff auf ausländische Domains zu sperren, welche es Swisscom-Kunden erlauben, im Ausland von Unbekannten urheberrechtswidrig verbreitete Inhalte in der Schweiz abzurufen. Abgesehen davon, dass auch illegal verbreitete Werke hinter der Eigengebrauchsschranke liegen und deshalb deren Konsum keine Urheberrechtsverletzung darstellt, fehlt es an einem adäquat kausalen Tatbeitrag der Swisscom.

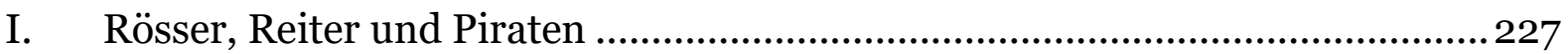

II. Problembeschrieb ................................................................................. 227

III. Erfolglose Einwendungen ....................................................................228

IV. Handlungsketten verbinden, aber binden nicht unbedingt ...........................228

V. Auch «Illegales» fällt unter die Eigengebrauchsschranke.............................229

VI. Nicht alles ist Teilnahme ....................................................................229

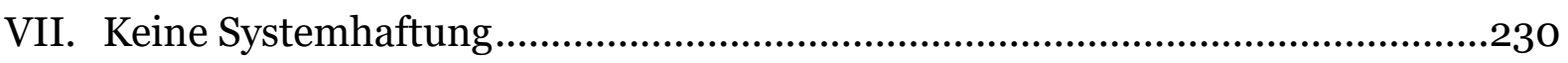

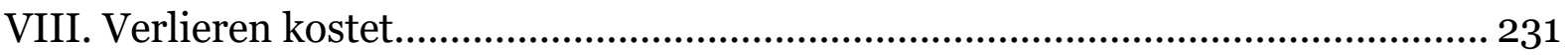

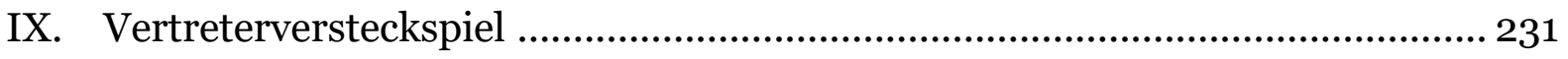

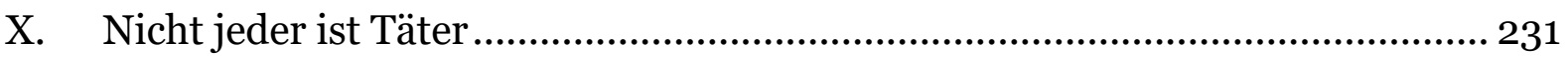

XI. Adäquanz schützt vor Zensur ...................................................................232

XII. Das Gejammer der Verlierer .................................................................233

Zitiervorschlag: Matthias Schwaibold, Das Bundesgericht weist die Filmverleiher in die Urheberrechtsschranken, in: sui-generis 2019, S. 227

URL: $\quad$ sui-generis.ch/105

DOI: $\quad$ https://doi.org/10.21257/sg.105

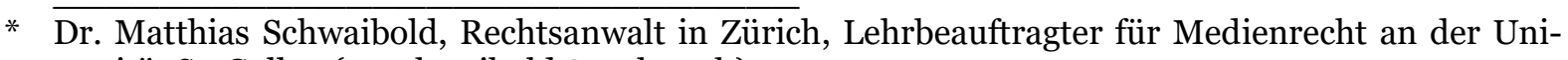
versität St. Gallen (m.schwaibold@rsplaw.ch).

Dieses Werk ist lizenziert unter einer Creative Commons Namensnennung - Weitergabe unter gleichen Bedingungen 4.0 International Lizenz. 


\section{Rösser, Reiter und Piraten}

1 «Illegal zugänglich gemachte Filme im Internet: Swisscom muss Domains nicht sperren. Die Swisscom kann als Access Provider (Anbieter von Internetzugang) nicht dazu verpflichtet werden, den $\mathrm{Zu}-$ griff auf Internet-Seiten mit illegal zugänglich gemachten Filmen zu sperren. Das Bundesgericht weist die Beschwerde eines Unternehmens ab, dem in der Schweiz die Urheberrechte für einen Teil der betroffenen Filme zustehen.»

2 Soweit die Einleitung der Medienmitteilung des Bundesgerichts zu seinem Urteil vom 8. Februar 2019, das seit dem 27. Februar 2019 über die Bundesgerichts-Website als 4A_433/2018 öffentlich ist; in der amtlichen Sammlung beschränkt sich BGE 145 III 72 auf die (lange) Erwägung 2.

3 Die Medienmitteilung nennt - im Gegensatz zum publizierten Urteil - die Beklagte, nämlich die Swisscom. Die Klägerin findet man, Internet sei Dank, selbstverständlich auch gleich heraus: Es ist die Praesens Film AG, die sich auf der Website der SAFE ( $\ll$ Swiss Anti Piracy Federation», zu deutsch «Schweizerische Vereinigung zur Bekämpfung der Piraterie») über das Urteil beklagt. Dazu später.

4 Warum das Urteil völlig richtig ist, soll nachfolgend zuerst aufgezeigt werden.

\section{Problembeschrieb}

5 Fassen wir zunächst die wesentlichen Erwägungen des Urteils zusammen:

6 Im Internet finden sich Portale, die Links auf andere Internetseiten enthalten. Die sogenannten «Hoster» erlauben es, Filme anzuschauen - entweder durch direktes Abspielen (Streaming) oder durch Herunterladen (Download). Die fraglichen ausländischen Portale finanzieren sich über Werbung und/oder Provisionen. Unbestritten ist das über die Portale vermittelte, von den Hostern zur Verfügung gestellte Material häufig insoweit «illegal», als weder die Hoster noch (unterstellt) diejenigen, die ihnen die Filme zugänglich machen, zu solcher (Weiter-) Verbreitung berechtigt sind. Insbesondere machte die Praesens Film AG, ohne dass wir vom Bundesgericht dazu Näheres erfahren, geltend, dass sie über die Exklusivrechte für das Gebiet der Schweiz betreffend einiger auf diese Weise zugänglicher Filme verfüge. Ihre vorgerichtliche Aufforderung, den Zugang zu bestimmten Portalen zu sperren, lehnte die Swisscom ab.

7 Mit Klage vor dem Handelsgericht des Kantons Bern verlangte Praesens, dass die Swisscom zu verpflichten sei, durch geeignete technische Massnahmen ihren Kunden den Zugang zu bestimmten Domains (mithin den vorgenannten Portalen) zu sperren. Das Handelsgericht wies die Klage ab, denn die Swisscom sei nicht Teilnehmerin an einer Urheberrechtsverletzung. Die Beschwerde der Klägerin ans Bundesgericht war erfolglos.

8 Dass die Klägerin ein schutzwürdiges Interesse hat und deshalb zur Beschwerde legitimiert ist, war - entgegen der Auffassung der Beklagten - nicht weiter fraglich; wie genau die Swisscom ihr Abwehrargument begründet hat, erfahren wir zwar nicht, aber es ist auch nicht ersichtlich, wie man diesen Standpunkt erfolgreich begründen könnte. Insoweit 
war auch klar, dass der Beschwerdeweg ans Bundesgericht offenstand. Das Bundesgericht wiederholt in Erw. 1.2. die Voraussetzungen, welche es an die Begründung einer Beschwerdeschrift stellt. Ebenso erinnert es in Erw. 1.3. daran, dass es seinem Urteil den von der Vorinstanz festgestellten Sachverhalt zugrunde legt, und es diesen Sachverhalt nur ausnahmsweise berichtigen oder ergänzen darf.

\section{Erfolglose Einwendungen}

9 Erster Beschwerdegrund war, dass die Vorinstanz die Passivlegitimation der Beklagten verneint hatte. Der Einwand erfolgte zu Unrecht (Erw. 2. bis 2.2.2.). Denn Passivlegitimation setzt gemäss Vorinstanz in unserem Zusammenhang zwei Dinge voraus: Eine Urheberrechtsverletzung eines Dritten (eine solche der Beklagten selbst wurde ihr richtigerweise nicht vorgeworfen!) und ein rechtlich relevanter Beitrag der Beklagten hierzu. Vom Rechtsbegehren nicht erfasst waren die «Hoster», also diejenigen, welche die fraglichen Filme zur Verfügung stellen.

Das Handelsgericht erinnert sodann an den Wertungsentscheid des schweizerischen Gesetzgebers: Veröffentlichte Werke dürfen zum Eigengebrauch verwendet werden. Das gilt auch dann, wenn die Quelle illegal ist. Mit anderen Worten: Eigengebrauch bzw. Werkverwendung im persönlichen Bereich stellt keine Urheberrechtsverletzung dar, auch wenn dabei Raubkopien oder in sonstiger Weise «illegale» Bezugsquellen ins Spiel kommen. Der Konsum der Filme über die fraglichen Domains ist keine Urheberrechtverletzung, welche die Endnut-
zer/Konsumenten/Kunden der Beklagten begehen.

11 Selbst wenn - fährt die Vorinstanz, immer gemäss Bundesgericht, fort - eine Urheberrechtsverletzung anzunehmen wäre, müsste die Beklagte einen rechtlich relevanten Beitrag dazu leisten. Die direkte Rechtsverletzung des Dritten müsse durch die Beklagte objektiv gefördert oder jedenfalls in allgemeiner Weise begünstigt werden. Indessen sei nicht jede Handlung eine solche Begünstigung, vielmehr wird ein «direkter Zusammenhang zur Tat» verlangt.

\section{Handlungsketten verbinden, aber binden nicht unbedingt}

12 Das Handelsgericht hat das mit einer «Handlungskette» veranschaulicht: Die beginnt bei demjenigen, der sich eine Kopie des urheberrechtlich geschützten Werks verschafft, setzt sich fort mit demjenigen, der diese Kopie auf einem Server speichert, es folgt derjenige, der den dafür nötigen Serverplatz zur Verfügung stellt, dann der Portalbetreiber, der das Auffinden des Filmes ermöglicht, und schliesslich derjenige, der den so gefundenen Film konsumiert. In dieser Handlungskette steht die Beklagte weit hinten (also ganz nahe am Konsumenten) und weit weg vom Verletzer. Die Dienstleistung der Beklagten ist nicht adäquatkausal für die widerrechtliche Haupttat und nach dem gewöhnlichen Lauf der Dinge und der allgemeinen Lebenserfahrung nicht geeignet, die fragliche Rechtsverletzung zu fördern. Damit fehlt es jedenfalls an der zweiten Voraussetzung des rechtlich relevanten Beitrags zur Verletzung. 


\section{Auch «Illegales» fällt unter die Eigengebrauchsschranke}

Seine eigenen Erwägungen beginnt das Bundesgericht damit, dass es daran erinnert, dass das Urheberrechtsgesetz (URG) im Gegensatz zu Design- und Patentgesetz keine Bestimmungen über Teilnahmehandlungen enthält. Aber selbst diese positiv-rechtlichen Bestimmungen in beiden Gesetzen gehen in der Sache nicht über das hinaus, was traditionell als Teilnehmerhaftung schon aus Art. 50 OR abgeleitet wird. Man kann Gehilfe einer Urheberrechtverletzung sein, ohne selbst einen gesetzlichen Verletzungstatbestand zu erfüllen. Eine analoge Anwendung der weitergehenden Bestimmungen des Persönlichkeits- oder des Sachenrechts wird abgelehnt. Die entsprechenden Erwägungen der Vorinstanz (die als solche zuvor indessen nicht erwähnt worden waren) werden als bundesrechtskonform erkannt. Anstiftung (im Sinne schuldhafter Veranlassung) liegt sowenig vor wie Begünstigung (also Vorschubleisten oder Fördern der rechtswidrigen Tat). Auch der Unterlassungsanspruch gegenüber dem Teilnehmer setzt voraus, dass die Urheberrechtsverletzung adäquat kausale Folge seines Beitrags ist.

Zudem setzt der Anspruch gegen den Teilnehmer eine rechtswidrige Haupttat voraus, hier also eine Urheberrechtsverletzung. Die Tätigkeit der Beklagten beschränkt sich darauf, ihren Kunden den Zugang zum Internet zur Verfügung zu stellen. Wenn der Kunde die streitgegenständlichen Internetseiten aufruft und die streitgegenständlichen Filme konsumiert, ist das unter dem Gesichtspunkt des Eigengebrauchs zulässig, «und zwar unabhängig davon, ob es sich um eine rechtmässig oder eine widerrechtlich zugänglich gemachte Quelle handelt». Unter Hinweis auf die Materialien zur letzten URG-Revision und zahlreiche Stimmen aus der Lehre kommt das Bundesgericht zum Schluss, dass das Herunterladen der Filme keine Urheberrechtsverletzung ist, an welcher die Beklagte hätte teilnehmen können. Die Vorinstanz hatte deshalb zurecht eine Urheberrechtsverletzung wie eine Teilnahme der Beklagten daran verneint. Mit der Bekräftigung des Standpunkts, dass der zulässige Eigengebrauch auch «illegal» erlangtes Material erfasst, dürfte das Bundesgericht eine weltweit wohl ziemlich einmalige Position zugunsten der Werknutzer eingenommen haben.

\section{Nicht alles ist Teilnahme}

15 Die Werknutzer verletzen also das URG nicht. Nicht geprüft hat die Vorinstanz Urheberrechtsverletzungen anderer Beteiligter, namentlich der Portalbetreiber, der «Uploader» und der Hoster; sie hat sich vielmehr in diesem Zusammenhang auf die Nutzer (= Kunden der Beklagten) beschränkt (Erw. 2.2.3. bis 2.3.). Die Klägerin behauptet vor Bundesgericht, sie habe ihre Rechtsbegehren gar nicht mit den Urheberrechtsverletzungen von Kunden begründet. Das kann ohne Blick in die Rechtsschriften vorliegend nicht überprüft werden, spielt aber auch keine Rolle: Denn es ändert am Problem nichts, dass der Beklagten eine rechtlich relevante Teilnahmehandlung an diesen Rechtsverletzungen Dritter (mit denen sie in keinen Vertragsbeziehungen steht) vorgehalten werden müsste.

16 Das Bundesgericht hat also zu prüfen, ob das Teilnahmeverhalten allgemein geeignet ist, die Urheberrechtsverletzung des 
Direktverletzers zu begünstigen. «Access Provider sind Zugangsdienstleister: Sie vermitteln Endnutzern den Zugang ins weltweite Internet.» Dieser Prozess läuft automatisch ab, also insbesondere ohne weitere manuelle Intervention des Access Providers, und dem Kunden wird dadurch der Abruf fremder (und das Hochladen eigener Daten) ermöglicht. Die Lehre ist in der Frage gespalten, ob der Beitrag des reinen Access Providers zu Urheberrechtsverletzungen adäquat kausal sei.

\section{Keine Systemhaftung}

17 Die Beklagte ermöglicht den Zugang zum Internet insgesamt, und es ist auch unbestritten, dass nicht sie selbst die fraglichen Datensätze mit den Filmen freigibt, sondern diese irgendwo in der Welt an unbekannten Orten zum Abruf freigegeben werden. Die Klägerin sieht den Beitrag der Beklagten darin, dass sie den Zugang zu den rechtsverletzenden Angeboten nicht sperrt. Als Access Provider bietet die Beklagte aber keine bestimmten Inhalte an, sondern ermöglicht den Datenaustausch ohne Bezug zu den Inhalten. Die Datenübertragung findet auf Abruf der Endkunden statt, die - wie vorstehend erklärt - gerade keine Urheberrechtsverletzung begehen (Eigengebrauch auch aus illegalen Quellen ist zulässig). Die technisch bedingte, vorübergehende Vervielfältigung der Filme ist gemäss Art. 24a URG vorliegend auch irrelevant. Access Provider haften, wie das Bundesgericht dann unter Hinweis auf weitere Bestimmungen festhält, nur als Haupttäter und nicht als Teilnehmer. «Weder die Portale, über die im Internet auf die fraglichen Filme zugegriffen werden kann, noch die Hoster, aber deren
Internetseiten die Filme direkt abgespielt werden, geschweige denn die Uploader, welche die Filme hochgeladen haben, sind Kunden der Beschwerdegegnerin.» Entgegen der Behauptung der Klägerin stellt die Beklagte diesen Dritten nicht ihre Infrastrukturleistungen zur Verfügung. Die Zugänglichmachung irgendwo im Ausland stellt die Rechtsverletzung dar, ohne dass hierfür die Beklagte einen konkreten (mithin rechtlich relevanten) Tatbeitrag liefern würde. Zugang zum Internet überhaupt zu verschaffen, reicht für eine zivilrechtliche Verantwortlichkeit als Teilnehmerin nicht aus. Das Bundesgericht verneint eine «Systemhaftung», die sich dann ja auf alle urheberrechtswidrigen Inhalte des Internet beziehen müsste, weshalb auch kein Anspruch auf technische Zugangssperren bestehen kann.

18 Mit seiner Entscheidung stellt sich das Bundesgericht gegen jenen Teil der Lehre, die eine Haftung der Access Provider befürwortet, und damit auch gegen eine entsprechende Entwicklung in anderen Staaten, die eine solche Haftung eingeführt haben. Die Frage des Für oder Wider ist hier nicht zu vertiefen - das Bundesgericht hat sich für die Schweiz festgelegt, und aus Sicht des Rezensenten ist sein Entscheid sowohl vom Ergebnis wie seiner Begründung her richtig; dass man das alles auch anders sehen kann, ist offensichtlich, darf aber vorliegend ausser Acht bleiben.

19 Der Entscheid ist auch problemlos mit dem Urteil des Bundesgerichts 5A_792/2011 vereinbar, in welchem die «Tribune de Genève» für einen Beitrag haftbar gemacht wurde, den jemand auf einem Blog publiziert hat, den sie auf ihrer In- 
ternetseite betrieb: Denn unter diesen Umständen war sie nicht etwa «Access Provider», sondern klarerweise «Hosting Provider»: «héberger un blog» bzw. «hébergeur de blog» zu sein, ist eben etwas entscheidend Anderes. Insoweit kann aus jenem Entscheid gerade nichts gegen den vorliegend zu besprechenden abgeleitet werden, im Gegenteil. Solange man richtigerweise zwischen Access Providern und Hostern zu unterscheiden hat, kann man nicht aus Verpflichtungen oder Haftungsbestimmungen, die für den letzteren gelten, einfach auf solche für den ersteren schliessen.

\section{Verlieren kostet}

20 Erfolglos war die Beschwerde auch bezüglich der Kosten- und Entschädigungsfolgen (Erw. 3.): Die Vorinstanz hatte angesichts des Aktenumfanges und der Komplexität der sich stellenden Rechtsfragen den jeweiligen Gebührenrahmen voll ausgeschöpft, was bei einem Streitwert von 75'00o CHF zu Gerichtskosten von 38'ooo CHF und einer Parteientschädigung von 42'0oo CHF, also Gesamtkosten von 80'0oo CHF für die Klägerin führte. Das Bundesgericht sah darin weder Ermessensmissbrauch noch Willkür, und es erinnerte daran, dass der Streitwert allein nicht massgeblich ist, wenn es darum geht, innerhalb solcher Gebührenrahmen den konkreten Fall auch kostenmässig einzuordnen. Die weitere Berufung auf die allgemeinen Verfahrensgarantien der Bundesverfassung (Art. 29) und der EMRK (Art. 6 Abs. 1) war nicht ausreichend begründet und erscheint auch dem Verfasser sinn- und zwecklos: Dass die Kosten- und Entschädigungsfolgen bei relativ niedrigen Streitwerten die Streitwerte selbst erreichen oder überschreiten können, ist auch unter diesen Gesichtspunkten nicht ausgeschlossen. Viel spannender wäre es zu wissen, wie hoch die Rechnungen der Anwälte an ihre Parteien gewesen sind.

\section{Vertreterversteckspiel}

21 Der Fall zeigt schliesslich, dass drei Anwälte auch nicht mehr erreichen können als einer - die Klägerin hat sich gemäss Bundesgericht durch drei Anwälte einer Anwalts AG vertreten lassen, die Beklagten durch einen einzigen einer anderen (sehr viel grösseren) Anwalts AG. Bemerkenswert, dass im Rubrum nur diese vier Anwälte, nicht aber die - wohl beauftragten und verantwortlichen! - Anwalts AGs als Vertreter bezeichnet sind.

\section{Nicht jeder ist Täter}

22 Wie eingangs erwähnt, ist dieser Entscheid in allen Teilen zutreffend begründet und kommt entsprechend zum richtigen Ergebnis. Nicht jedes Delikt eines Dritten begründet Ansprüche gegenüber solchen, die in der «Handlungskette» zwischen Deliktsbegehung und Deliktserfolg irgendwo anzusiedeln sind. Das Handelsgericht hat diese Handlungskette (wohl nicht ganz vollständig) aufgezeigt: Zwischen dem Konsumenten, der in der Schweiz via Internet einen Film ansieht, und jenem (jenen) Unbekannten im Ausland, die diese Filme ins Internet stellen, gibt es eine Reihe Dritter, von denen zumindest die Hoster und die Access Provider technisch unabdingbar sind, vorliegend aber noch durch weitere Zwischenstationen (insbesondere die in der Klageschrift genannten Portale) voneinander getrennt sind. In der konkreten Kausalkette zwischen den «Uploadern» 
und den «Usern» verdünnt sich - rechtlich gesehen - der Tatbeitrag eines jeden, je weiter er vom Verletzer entfernt ist. Geht man mit Handels- und Bundesgericht davon aus, dass jedenfalls der User/ Nutzer/Konsument in der Schweiz keine Urheberrechtsverletzung begeht, sondern die am «anderen» Ende der Kette (also beim «Uploader») zu suchen und zu finden ist, dann ist es auch klar, dass man dem blossen Access Provider weder eine Teilnahmehandlung vorwerfen noch eine daraus abgeleitete Unterlassungsverpflichtung auferlegen kann.

Abgesehen davon stellt sich die Frage, ob die hier verlangte «Unterlassung», nämlich die Sperrung bestimmter InternetDomains (insb. der ausländischen Vermittlungsportale) nicht eher eine Handlungspflicht ist. Damit würde - vermutlich - auch eine Vertragsverletzung gegenüber dem eigenen Kunden begangen werden: Der hat Anspruch darauf, auf alles zugreifen zu können, was das Internet anbietet, und nur dort, wo aufgrund eindeutiger gesetzlicher Bestimmungen (z.B. Kinderpornografie) der Endkunde durch den Konsum sich strafbar macht, könnte man seinem Access Provider allenfalls eine adäquat-kausale Teilnahme vorhalten. Dazu kommt, dass es nicht nur in der Schweiz, sondern weltweit Access Provider gibt, mithin das Problem der Rechte-inhaber nicht gelöst ist, wenn ein Access Provider die $\mathrm{Zu}-$ gangssperren errichtet. Zurecht fällt seitens des Bundesgerichts der Ausdruck «Systemhaftung», und da es die nun einmal nicht gibt, war auch der klägerische Anspruch zum Scheitern verurteilt. Das gilt nicht nur dann, wenn man «rückwärts» vom Endkunden und Filmkonsumenten in der Schweiz via
Swisscom zum «Uploader» zurückdenkt, sondern auch «vorwärts» von diesem: Die Swisscom stellt ihm ihre Infrastruktur gerade nicht zur Verfügung, sondern er profitiert davon, weil ihr Netz eines von vielen technisch nötigen, untereinander verknüpften Netzen ist - das allein auf Veranlassung des Endkunden aber überhaupt erst ins Spiel kommt.

\section{Adäquanz schützt vor Zensur}

24 Internet und territoriale Beschränkung von Verbreitungsrechten stehen in einem gewissen inneren Widerspruch; dass das Urheberrecht sowohl de lege lata wie de lege ferenda zunehmend dazu dienen soll, Verwertungsmöglichkeiten zu schaffen bzw. zu schützen, wobei die Nutzniesser nicht immer die im eigentlichen Sinne geistigen Urheber oder Werkschöpfer sind, lässt sich auch nur bedauern, aber der Trend kaum umkehren. Umso wichtiger ist es, dass das geltende Recht nicht dazu missbraucht wird, um es deutlich zu sagen, jeden, der irgendwo technisch unvermeidlich an einer Urheberrechtsverletzung beteiligt ist, zum Täter oder wenigstens Teilnehmer zu machen und ihm Handlungs- resp. Unterlassungspflichten aufzuerlegen. Wer lediglich die Infrastruktur zur Datenübertragung zur Verfügung stellt, wer insbesondere seinem Kunden nur den Zugang zum Internet ermöglicht, aber nicht mehr macht, der leistet zwar einen kausalen, aber keinen adäquat-kausalen Beitrag zur Urheberrechtsverletzung, die Dritte begehen. Um einen etwas gewagten Vergleich zu machen: Auch Alleinvertriebssysteme und die entsprechende Preisbindung versuchte man vergeblich zu schützen, indem man aus dem Rechtsbruch (unbekannter) Dritter 
Pflichten derer abzuleiten versuchte, die von diesem Rechtsbruch profitierten (namentlich Unterbindung von Parallelimporten). Hier profitiert die beklagte Swisscom jedenfalls nicht in ersichtlicher Weise davon, dass andere Urheberrechte verletzen, wobei es unter diesem Gesichtspunkt nicht einmal darauf ankommt, dass nach geltendem Recht die Vertragspartner der Swisscom (Endkunden/ User/Nutzer/Filmkonsumenten) gar keine Rechte verletzen. Selbst wenn man in Verkennung oder nach formgerechter Abänderung der Eigennutzungsschranke plötzlich auch den Filmkonsumenten zum Verletzer machen würde, wäre damit noch nicht begründet, dass auch der Access Provider einen adäquat-kausalen Tatbeitrag leistet, denn trotz der (unmittelbaren) Nähe zu diesem SekundärVerletzer (wenn wir ihn damit von «Primärverletzern» - in der Diktion des Bundesgerichts «Direktverletzern» - wie den Uploadern oder deren Hoster unterscheiden wollen) wäre die blosse $\mathrm{Zu}$ gangsvermittlung weder ein Fördern noch ein Begünstigen oder dergleichen, sondern lediglich ein (technisches) Ermöglichen. Indessen haftet auch nicht der Autohersteller oder -verkäufer für das Raserdelikt, sowenig wie der Werkeigentümer der Strasse. Man mag alle diese Vergleiche für gewagt, weit hergeholt oder gar untauglich halten, allemal geht es darum, eine Botschaft zu vermitteln: Absolute oder Monopolrechte dürfen sowenig wie relative Rechte voraussetzungslos zu Ansprüchen gegenüber irgendwelchen Dritten führen. Teilnahmeerfordernisse müssen sich streng an dem ausrichten, was man aus Art. 50 OR ableiten kann, und die Adäquanz als rechtspolitische Einschränkung der natürlichen Kausalität darf nicht einfach dem Schutz von Rechteinhabern geopfert werden.

25 Dass weltweit betrachtet die Verpflichtung zu Netzsperren auf dem Vormarsch ist, heisst noch lange nicht, dass das Bundesgericht auf dem Holzweg ist: Ein liberales Rechtsstaatsverständnis geht nun einmal davon aus, dass der Staat nicht einfach voraussetzungslos der Vollstrecker jedes privatrechtlichen Anspruchs sein muss. Weshalb auch nicht einfach jeder in einer «Handlungskette» zum Störer, Mittäter, Gehilfen oder Verletzer gemacht werden darf. Und letztlich muss man sich auch, so platt das klingen mag, Geist und Buchstabe des Zensurverbots vor Augen halten, die allen Netzsperren zunächst einmal doch entgegenstehen. So gesehen, steht der Entscheid in Einklang mit tragenden Grundsätzen unserer Rechtsordnung.

\section{Das Gejammer der Verlierer}

26 Womit ich - wie eingangs angekündigt unter zwei Gesichtspunkten auf SAFE zurückkommen möchte: Die «Schweizerische Vereinigung zur Bekämpfung der Piraterie» begeht unlauteren Wettbewerb allein deshalb, weil «Piraterie» nur das ist, was auf See stattfindet - sie bekämpft nur, aber immerhin «Internet-Piraterie» oder «Urheberrechts-Piraterie». Aber auch noch aus einem zweiten Grund sollte man diesem Verein die Leviten lesen: «Legale Angebote im Internet weiterhin ungeschützt: Rechteinhaber gehen vor Bundesgericht». Das war der Titel der SAFE-Mitteilung über das erstinstanzliche Urteil. Unverständlicher geht es kaum noch - denn was ist jetzt «ungeschützt», und warum gehen die Rechteinhaber vor Bundesgericht? Ein korrek- 
ter Titel hätte zum Beispiel ganz einfach gelautet: «Illegale Angebote im Internet weiterhin geschützt: Rechteinhaber gehen vor Bundesgericht». Das wäre glasklar gewesen. Auch der Untertitel dieser Meldung ist nicht eben redlich: «Gemäss Urteil vom Berner Handelsgericht können Access Provider weiterhin Zugang zu illegalen Seiten gewähren.» Nein, gemäss dem Urteil sind sie nicht verpflichtet, den Zugang zu sperren, wie es verlangt worden war. "Gewähren dürfen» ist nun wirklich nicht dasselbe wie «nicht sperren müssen». Aber auch die Meldung zum Bundesgerichtsurteil ist Desinformation: «Bundesgericht schwächt Schutz des Urheberrechts». Auch hier wird der Leser in die Irre geführt, denn das Bundesgericht hat nicht einen bestehenden Schutz geschwächt, denn «Schwächung» setzt Existenz voraus, sondern die Behauptung eines Schutzes (um den Ausdruck «Schutzbehauptung» zu vermeiden) als nicht begründet erachtet. Das ist nun einmal nicht dasselbe. Dass «der Schutz von Urheberrechten vor schweizerischen Gerichten, in Ermangelung klarer Verantwortlichkeitsregeln, ein schwieriges Unterfangen» sei, ist die letzte Behauptung, die ich hier auf- und angreifen will: Die Verantwortlichkeitsregeln waren und sind klar. Sie gehen einfach nicht soweit, wie es der Klägerin und dem sie stützenden Branchenverband lieb wäre. Das ist indessen hinzunehmen. 